\title{
Comparative Aspects of Nanoparticles in Relation to Extending Shelf-Life of Molds
}

\author{
Jan Novotný1*, Irena Lysoňková', Jaromír Cais', Štefan Michna' \\ 1 Faculty of Production Technology and Management, J.E. Purkyne University in Usti nad Labem, Pasteurova \\ 3334/7, 40001 Usti nad Labem, Czech Republic \\ * Corresponding author's e-mail: novotny@fvtm.ujep.cz
}

\begin{abstract}
The paper deals with the problems of nanoparticles and nanomaterials applied in technical practice. The research on the use of nanoparticles has at present a great development. Their potential has a large of use. Particles of nanosize, i.e. below $100 \mathrm{~nm}$ exhibit other properties than larger particles and because this area is being explored in all possible sectors. The effect of adding these particles at different mechanical properties in coatings on various surfaces neat (separated on a band saw, milling work, ground roughness 800 and 80 polished) dependent on quality prepared nanoparticles by different conditions of milling. One of the possibilities for an extension of the duty cycle of tyre production molds is applying a protective coating. Due to the functionality of the desktop is needed a very thin coating layer. A suitable type of coating is thus a PTFE coating, which prolongs the service life of hundreds of percent. Better possibility how to improve an extension of the duty cycle is the addition of the coating titanium dioxide particles into the PTFE medium. This is the way how to create nanocomposite directly on the mold surface.
\end{abstract}

Keywords: nanoparticles, engineering practice, nanocomposites, aluminum alloys.

\section{INTRODUCTION}

Samples evaluated in this article are prescribed and evaluated in order to prolong the life of molds. Evaluation aspect was determined as the surface roughness of the working surface of the mold. Evaluation homogeneity of nanocomposite was assessed by scanning electron microscopy. In this article there is a research added nanoparticles namely titanium dioxide into polytetrafluoroethylene nano-coatings, which are applied on the working area used for injection molding of rubber compounds. The purpose of this addition is to increase the strength and durability of said nanocoating.

For the mold production are used aluminum alloys without any surface modification, that is casted under pressure. Since maintenance between successive cycles is costly, there is an effort to extend those cycles. The number of working cycles is usually between 2500 and 2700 . Due to attempting of the extension of the working cycles is applying of PTFE coat onto the surface.
PTFE coating leads to increasing of the number of cycles from 200 to $400 \%$ [7].

Good choose for life extension of used molds can be by application of nanocomposite directly on the surface of the mold.

\section{INJECTION MOLD}

Mold used for injection molding of polymer materials (in this case tires of different types) consist of 8 to 36 segments according to the size and application one of the segments of an injection mold is shown in Figure 1.

The segments are produced by low pressure casting of aluminum alloy of the Al-Si. Specifically, the alloy type AlSi10CuNiMn. Use of this type of coating is also possible for alloys Al-Mg. The proposed composition and subsequently the specific alloy composition was evaluated on the spectrometer Q4 TASMAN. Results are in Table 1. Usage of thus alloys is appropriated due high performance proper- 


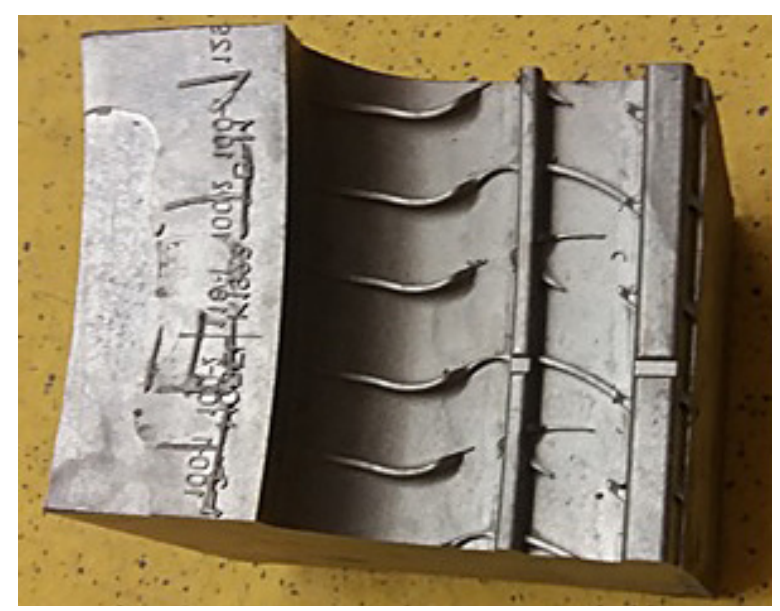

Fig. 1. Segment mold for tires production casted from aluminium alloy

ties at normal and elevated temperatures. It is necessary to prove stable mechanical properties of mold when works under elevated temperatures. Tires are produced at a temperature of $150-170^{\circ} \mathrm{C}[5]$.

\section{MOLD COATING}

Coating of molds by the PTFE is carried out by the following procedure:

- detoxification - rinsing in demineralised water

- coating I phase - rinsing with demineralized water - drying.

- coating II.phase - rinsing with demineralized water - drying.

This type of coating is incorporated into nano- coatings and due to its thickness does not affect properties of the product itself. Prices molds are in the hundreds of thousands to millions of crowns and therefore we should continue to explore other possibilities for life extension. In this case the effort to create the nanocomposite coating of PTFE - $\mathrm{TiO}_{2}$.

\section{POLYTETRAFLUORETHYLENE COATING}

This type of coating is belonged to nanocoating, also because we tried implement to it nanoparticles. This coating is often referred as Teflon.
The coating technology is used for coating aluminium forms from material $\mathrm{Al}-\mathrm{Si}$ and $\mathrm{Al}-\mathrm{Mg}$. In this case was used alloy with Aluminum and Silicone. These materials are for the production of tires in the auto move industry.

Procedure effort the coating is as follows: pickling - rinse in demineralised water - coating I. phase - rinse in demineralised water - drying - coating II. phase - drying. Alone coating extends the possibility to use without purification of $200-400 \%$, which reduced wear of the mold cleaning [7].

The main components of the coating are $\mathrm{C}, \mathrm{O}$, F. ON the Figure 2 is nanocoating without addition titanium dioxide. If we add particles so it is in II. phase. It is achieve further increased durability. Powder was simply thrown into the coating and stirred manually. Any increase in life forms reduces manufacturing costs.

\section{COATED MATERIAL}

Used material is aluminium alloy of type Al$\mathrm{Si}-\mathrm{Cu}$, specifically AlSi10CuNiMn. This alloy is used for low-pressure casting. Its advantage is the possibly to use at higher temperatures, which exhibits stable mechanical properties. Because this research relates specifically to the processing of polymer material, nay indicate a temperature from 150 to $170^{\circ} \mathrm{C}$. The melt for the formation of each segment is at the casting temperature mained in the casting furnace, and therefore requires even the temporal stability of the melt [5].

\section{TITANIUM DIOXIDE AND ITS NANOPARTICLES}

Titanium dioxide $\left(\mathrm{TiO}_{2}\right)$ is widespread and the comonly used substance. It is known as titanium dioxide and is used for example as a colorant. It has many useful features such as UV rezistentnost (use sunscreen), a high refractive index, etc. Nowadays we are looking for an exploring ways how to make best use of the properties of the material.

Table 1. Recommended and real content of used alloy

\begin{tabular}{|c|c|c|c|c|c|c|}
\hline Element & $\mathbf{S i}$ & $\mathbf{C u}$ & $\mathbf{N i}$ & $\mathbf{M n}$ & $\mathbf{M g}$ & $\mathbf{S r}$ \\
\hline Recomended content [\%] & $8.5-10$ & $0.6-1.2$ & $0.6-1.0$ & $0.3-0.7$ & $0.3-0.5$ & $0.03-0.005$ \\
\hline Real content [\%] & 10.7 & 1.02 & 0.98 & 0.31 & 0.56 & 0.032 \\
\hline
\end{tabular}


When applied on the base material (metal, plastic, glass) of a very thin layer of a different material, will obtain this base material very different properties. Most of the thus obtained properties is very advantageous and improves mechanical properties such as corrosion resistance, hydrophobicity, non-attachment of dust on the surface and the like. To obtain these properties, it is necessary to apply this layer of fine nanoparticles (units of nanometers to hundreds of).

\section{SAMPLES FRACTOGRAPHY}

Only the finest fraction of $\mathrm{TiO}_{2}$ from the ground sample is taken for the purpose of electron microscope analysis of the grain morphology. For nanocomposite coating is used the smallest grain fraction from sample, after the first fine grinding From the geometry of the grains it is obvious that we are in the nanometer range. The shape of the grains is irregular with sharp edges. This is typical for the mechanical ball mills grinding. That is probably reason of a large grain aggregation. Aggregation is also caused by charge on the grain surface. With grain size decreasing, relative surface forces are growing. They impede the classical gravity (vibration) screening. From visualization it is apparent, that even these methods can produce a nanometres sort of particle range.

The above mentioned procedures persuaded us, that we are able to prepare $\mathrm{TiO}_{2}$ nanometer size fractions. There are very important suitable input parameters, which are affecting subsequent milling process. Any change of input parameters has a major influence on the milling process. Thus we achieved good yields of re - milled powder. This process is very sensitive on entrance variable and is more difficult to compare it with the first milling. As it turned out, suitable is the same milling process as used in the previous case. Based on images from the electron microscope, we achieved very satisfactory results in terms of grain sizes. As we found out, in this case we can hardly use vibration sieving machine for selection of nanopowder, due to strong aggregation of the particles. The solution can be the usage of different method of distribution of fraction sizes or direct usage of milled powder without sieving. But this procedure can be applied in the case that the subjected sample is composed namely from grains of very fine fractions.

\section{FORMATION OF NANOCOMPOSITE COATING}

The addition of nanoparticles was carried out in II. phase coating. However, the first solution of the problem, the quantity of applied powder. In the first phase they were used in concentrations of $2 \%, 5 \%$ and $9 \%$. Results of evaluation on the scanning electron microscope were satisfying, however, during a coating operation, there was considerable adhesion of large amount of particulate on the bottom of the coating vessel even when an attempt dispersion and mixing glass stick.

In the second stage coating was used a magnetic stirrer, which was completely disperse the particles in a coating solution, all due to the large amount of particles became the samples not rate. The layer in this case was in the order of millimeters.

Due to this extreme amount was allowed to use the same technology of electromagnetic stirrers, but with concentrations of $0.5 \%, 0.05 \%$, $0.01 \%, 0.005 \%$ and $0.001 \%$. To verify the suitable concentrations was used a scanning electron microscope TESCAN Vega 3 EDS analyzer equipped with a Bruker X-FLASH. As the most suitable concentration revealed $0.01 \%$. At lower concentrations were detected significantly smaller amount of particles, and at higher concentrations caught noticeable to the naked eye layer.

Images with uniform capture and identification are listed below. It is visible the surface analysis was performed using a scanning electron microscope. Figure 6 shows the result of analysis where are the individual elements contained therein paeks. From the analysis it can be determined that aluminum, silicon, magnesium is present in the substrate. Oxygen and fluorine are contained in PTFE coating. Titan is part of the additives to improve coating.

\section{EXPERIMENT AND ITS EVALUATION}

First measuring is the roughness on the desktop of one segment forms. Samples were prepared in the form of a plate of $35 \mathrm{~mm} \times 45 \mathrm{~mm}$ and with different finishes machined by: cutting, milling, grinding and polishing.

To the coating medium for samples coating were added $\mathrm{TiO}_{2}$ particles of during the second. phase of coating process. $\mathrm{TiO}_{2}$ particles were added the percentage content of $0 ; 2 ; 5$ and $9 \%$ rela- 
Table 2. Roughness of milling surface coated by various concentrations in $\mathrm{TiO}_{2}$ - PTFE nanocomposite

\begin{tabular}{|c|c|c|c|}
\hline Specification & $\mathbf{R a}$ & $\mathbf{R z}$ & $\mathbf{R t}$ \\
\hline Work area & 4.13 & 22.71 & 34.83 \\
\hline Coating $0 \% \mathrm{TiO}_{2}$ & 2.96 & 12.65 & 14.81 \\
\hline Coating $2 \% \mathrm{TiO}_{2}$ & 2.77 & 12.30 & 15.26 \\
\hline Coating $5 \% \mathrm{TiO}_{2}$ & 1.90 & 9.52 & 12.69 \\
\hline Coating $9 \% \mathrm{TiO}_{2}$ & 2.18 & 10.84 & 13.79 \\
\hline
\end{tabular}

tive to the particle mass ratio of coating medium. During process of coating begin to realize of aggregation of particles on the bottom of vessel. It is necessary to use suitable mixing device.

Samples of identical roughness are mentioned by those which have surface machined by milling. The values are in Table 2. The roughness was measured on the HOMMEL Tester T8000 according to EN ISO 4287.

Particles of $\mathrm{TiO}_{2}$ did not have the desired particle size. Thus particles were milled particles in the planetary ball mill PM 100 Retsch. After milling process the particles reach a size of the "nano" part i.e. below $100 \mathrm{~nm}$. We are in this stage of research concentrated on the grain size of the order of $100 \mathrm{~nm}$.

Working with very small particles is complicated due to possible clustering of particles by the influence of electrostatic forces. Other evaluation parameter was to assess the homogeneity of particles distribution in PTFE matrix by the scanning

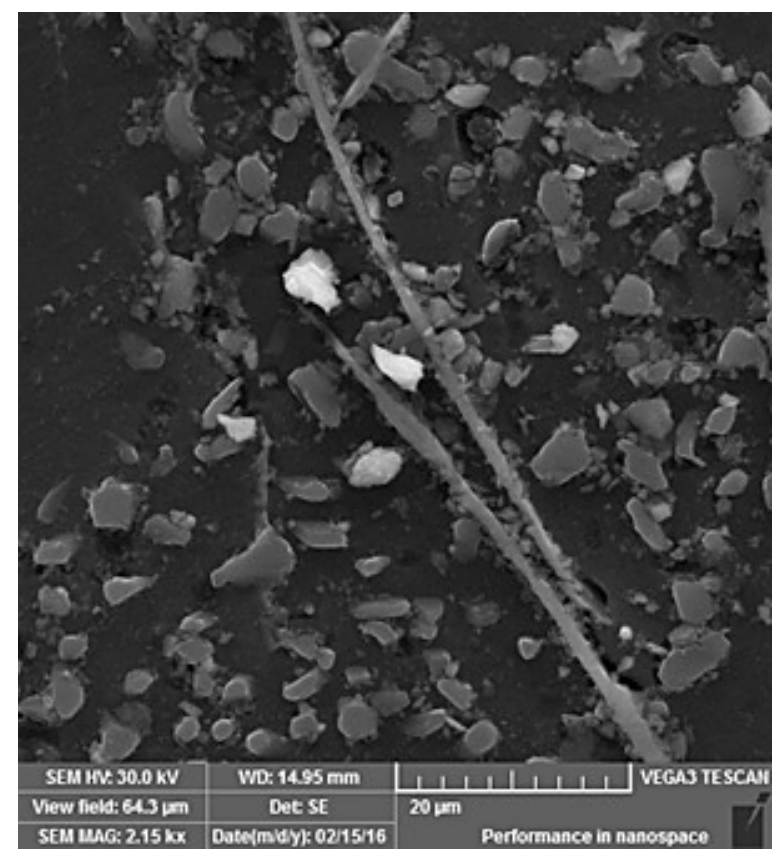

Fig. 2. $\mathrm{TiO}_{2}$ particles distribution in PTFE matrix

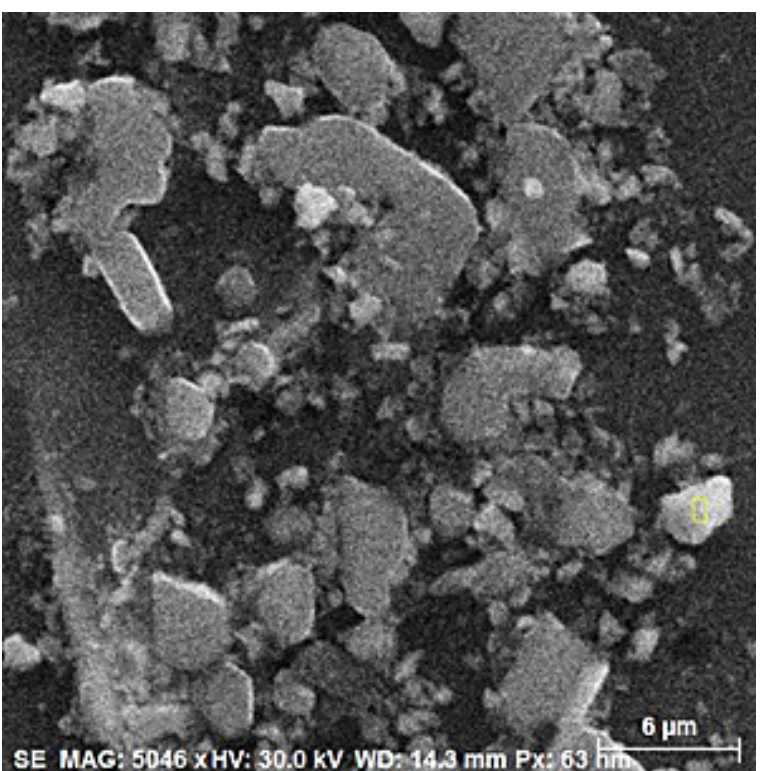

Fig. 3. EDS analysis - marking of evaluated particle 1

electron microscope TESCAN Vega 3 equipped with a Bruker EDS analyzer.

We can observe a uniform distribution of particles of $\mathrm{TiO}_{2}$ (in Fig. 2). It is possible to talk about the successful creation of nanocomposite technology assessment, where are no anticipated clusters.

Evaluation of $\mathrm{TiO}_{2}$ content in the matrix is in FIG. 3. Results are evaluated by EDS analysis (Fig. 4; Table 3). The aim of analysis of the exact chemical composition is based on of the surface analysis. In this case, for the evaluation was used milled sample. Content of $\mathrm{TiO}_{2}$ particles in PTFE matrix is $9 \%$ relative to the amount of PTFE macromolecules. From the chemical composition can be determined that aluminum, silicon and magnesium are the base material. Titanium and oxygen

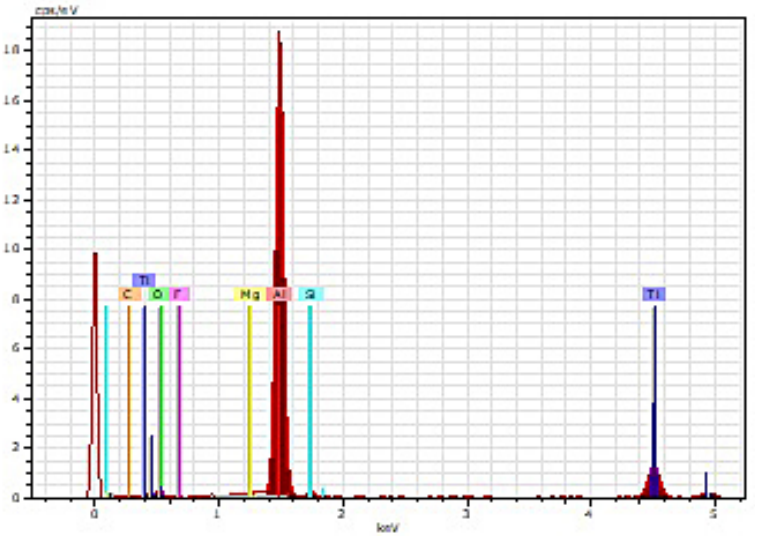

Fig. 4. EDS analysis - chemical composition of particles 1 
Table 3. EDS analysis - mass and atomic concentration of particles 1

\begin{tabular}{|c|c|c|}
\hline Element & $\begin{array}{c}\text { Mass } \\
\text { concentration [\%] }\end{array}$ & $\begin{array}{c}\text { Atomic } \\
\text { concentration [\%] }\end{array}$ \\
\hline Aluminium & 62.03 & 52.89 \\
\hline Oxygen & 13.53 & 19.45 \\
\hline Titanium & 10.43 & 5.01 \\
\hline Silicon & 1.78 & 1.46 \\
\hline Fluoride & 2.08 & 2.52 \\
\hline Magnesium & 0.81 & 0.76 \\
\hline Carbon & 9.35 & 17.91 \\
\hline
\end{tabular}

Table 4. EDS analysis - mass and atomic concentration of particles 2

\begin{tabular}{|c|c|c|}
\hline Element & $\begin{array}{c}\text { Mass } \\
\text { concentration [\%] }\end{array}$ & $\begin{array}{c}\text { Atomic } \\
\text { concentration [\%] }\end{array}$ \\
\hline Aluminium & 65.99 & 52.89 \\
\hline Oxygen & 16.64 & 22.48 \\
\hline Silicon & 3.15 & 2.43 \\
\hline Fluoride & 1.54 & 1.75 \\
\hline Titanium & 1.26 & 0.57 \\
\hline Magnesium & 0.75 & 0.66 \\
\hline Carbon & 10.67 & 19.22 \\
\hline
\end{tabular}

comes from the $\mathrm{TiO}_{2}$. Fluorine, oxygen and carbon comes from the PTFE coating.

On the Figures 5 and 6 we can identify too particles of titanium dioxide with EDS analysis. This particle is marking with cross. On this

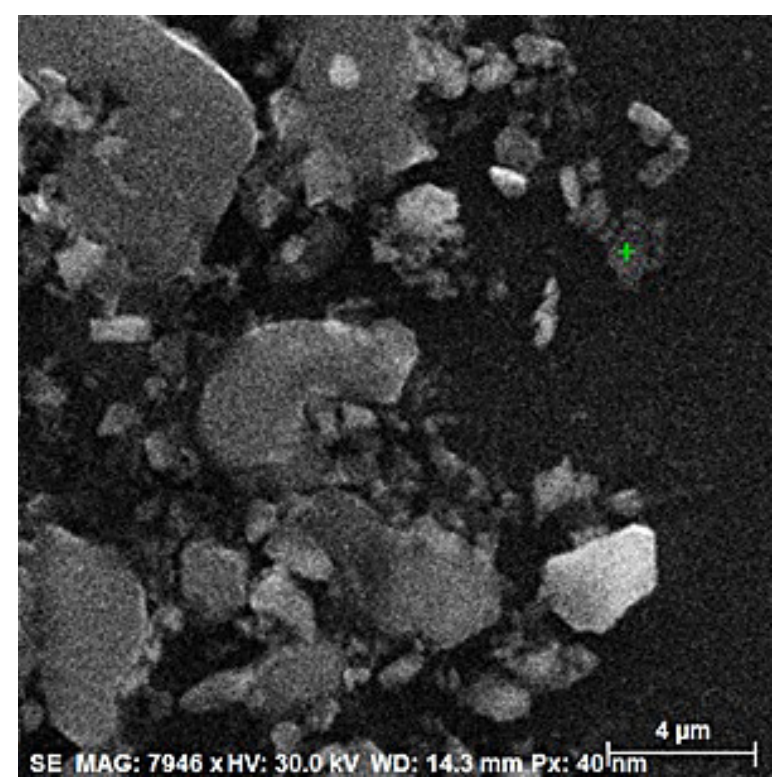

Fig. 5. EDS analysis - marking of evaluated particle 2

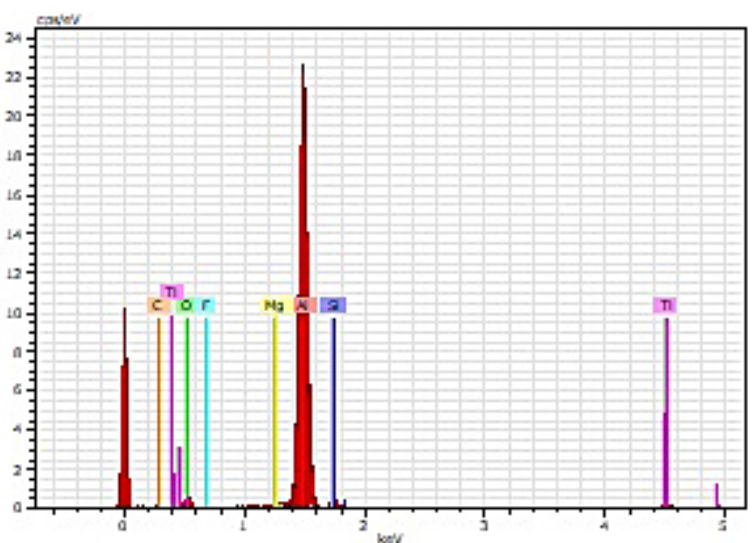

Fig. 6. EDS analysis - chemical composition of particles 2

place we identify (Table 4) in substrat aluminium, silicon and magnesium. Next in coating oxygen, fluorine and carbon. Titanium proves its existence in the coating. On Figures 7 and 8 we can see chemical element map. This is important because it shows a uniform layout of titanium.

\section{CONCLUSIONS}

The aim of the work was to find the possibility of extending the lifetime of working surface molds. Next goal was to find optimum conditions and analysis of the grains resulting surfaces.

By the experimental activity succeeded in creating nanocomposite material - PTFE and

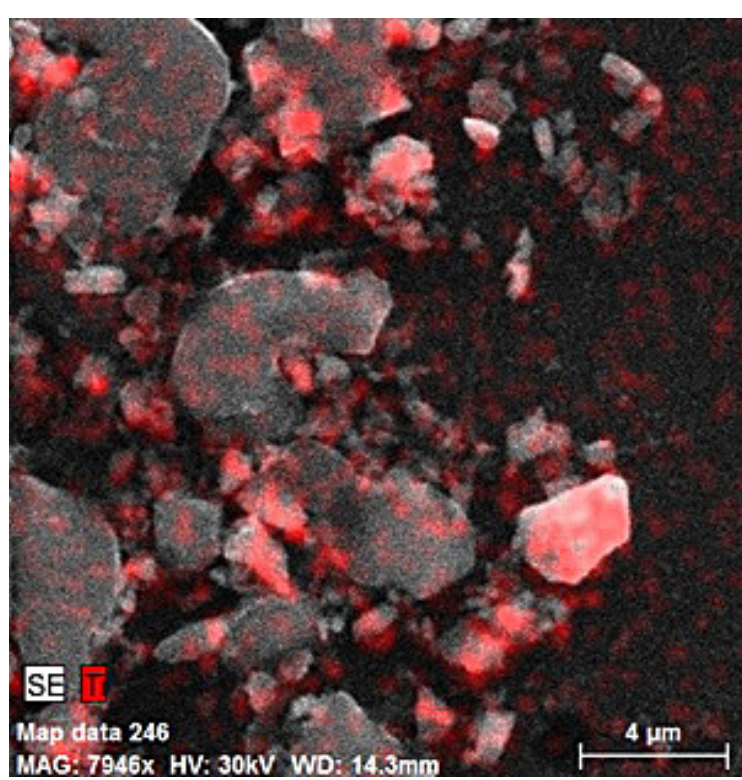

Fig. 7. EDS analysis - chemical element map of particles 2 


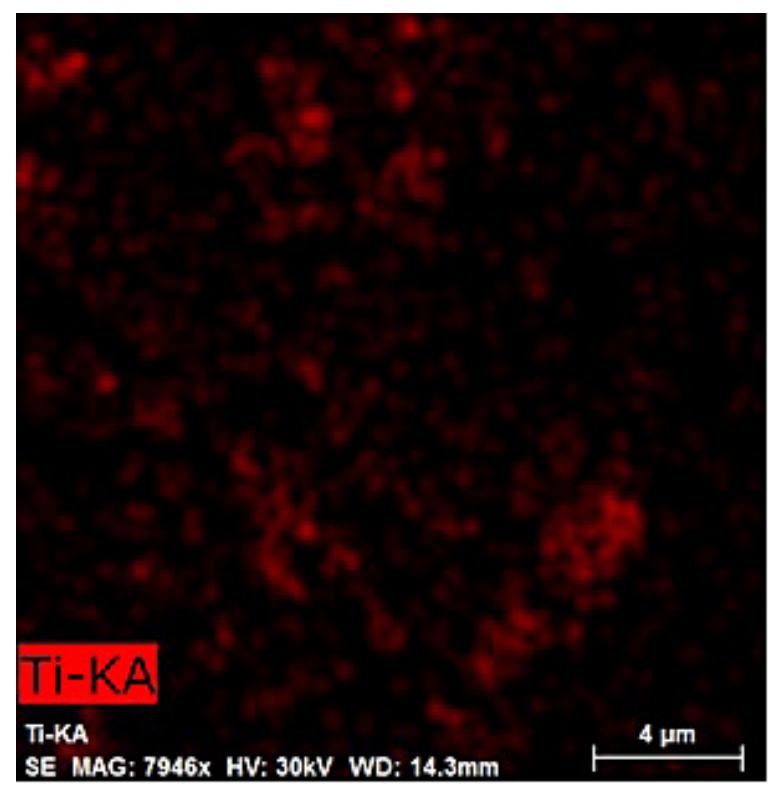

Fig. 8. EDS analysis - chemical element map of particles 2

$\mathrm{TiO}_{2}$. Evidence of the formation of this nakompozitu EDS analysis, which confirmed the content and uniform distribution of titanium dioxide. Due to the establishment of large quantities of titanium dioxide coating to the bottom of the vessel is suitable for further experiment used a combination of a smaller quantity of titanium dioxide and mixing equipment. 3. Using electron microscopy identified particle of titanium dioxide, basic material and coating. On the figure we can see biggest particles and nanoparticles. Regarding can also be, that one is particle at the surface and other are directly in the coating, what is desirable. Particles in solid are mixed by hand, causing sticking on the bottom. Next adequate outcome is suitable mixing device.

\section{Acknowledgements}

This article was created due to the project nr. 4820215001001 ,SGS“"under the specific university research on FVTM JEPU.

\section{REFERENCES}

1. Cais, J., Žihalova, M., Bolibruchova, D.: Microstructural Analysis of Nickel Influence in AlSi10MgMn Alloy with Increase Iron Level. Manufacturing Technology, 15(4), 2015, 743-748.

2. Giacovazzo, C.: Fundamentals of Crystallography. Oxford University Press, USA 2002.

3. Kejzlar, P. Structure and mechanical properties of $\mathrm{Fe}-25 \mathrm{Al}-5 \mathrm{Zr}$ and $\mathrm{Fe}-30 \mathrm{Al}-5 \mathrm{Zr}$ intermetallic alloys. Manufacturing Technology, Vol. 12, 2012.

4. Lysoňková, I., Novotný, J., Cais, J., Michna, Š.: The Effect of Addition of Nanoparticles TiO2 into PTFE Coating. Engineering for Rural Development, Vol. 16, 2017.

5. Michna, Š., Cais, J. Aluminum alloy in particular for the production of mould segment for forming typesand the method of heat treatment of mould segment casting. CZ/28.07.15/CZA 20150521.

6. Novotný, J., Jaskevič, M.: Morphology and producing of $\mathrm{TiO} 2$ particles, Engineering for Rural Development, Vol. 15, 2016, 1218-1223.

7. Standard 13420/13:43885462. Zavedení technologie chemického povlakování $\mathrm{Al}$ forem za vznik ochranných nanovrstev.

8. Valašek, P., Muller, M.: Polymeric particle composites with filler saturated matrix. Manufacturing Technology, Vol. 12, 2012.

9. Vít, M., Kotlík, B.: Zdravotní aspekty užití nanočásticvčetně nástřiků s TiO2. SZU Praha.

10. Weiss, V., Strihavková, E.: Influence of the homogenization annealing on microstructure and mechanical properties of $\mathrm{AlZn} 5,5 \mathrm{Mg} 2,5 \mathrm{Cu} 1,5$ alloy. Manufacturing Technology, Vol. 12, 2012. 